\section{Sharing different mouse strains is not always so simple}

SIR - I was disappointed by the view expressed in your Editorial 'The sharing principle' (Nature 459,752 ; 2009) that the mouse community does not share its strains. This is untrue. Most labs are very collegial, spending a considerable amount of time and effort on distributing their mouse strains. Although there are a few labs that withhold distribution, any community may contain such individuals. The fact that a mouse strain is not found in a repository does not mean that it is not being shared.

I was also puzzled by the conclusion of May's CASIMIR workshop, noted in the Editorial, that "the sharing problem urgently needs resolution" with regard to international mouse geneknockout projects. Such mutant alleles will mostly be archived as embryonic stem-cell lines. Readers should also realize that repositories cannot keep all their mouse strains live 'on the shelf': most strains are frozen. The cost for a user to have a strain thawed is thousands of dollars and it takes many months before the recovered mice become available. This is a big disadvantage for labs on tight budgets. With regard to funding agencies: in grant proposals to the US National Institutes of Health, for example, applicants are required to write a 'resource-sharing plan' that includes genetically modified mice.

It was suggested that sharing avoids duplication of effort. But it is essential that more than one group generates mutations in the same gene as a crosscheck. No two labs generate the same allele, and every geneticist knows that the expression of different alleles can lead to very distinct phenotypes.

Your claim that sharing mice "has never been easier" is questionable, considering all the paperwork, health certificates, veterinary screens, special serology screens, costs, time and logistics involved. This is quite different from uploading DNA sequences in the comfort of your office.

It would be great if funding agencies supplemented grants involving the generation of mouse strains to cover the costs of sending the strains to a repository. In these tough financial times, that seems unlikely.

Richard Behringer Department of Genetics, University of Texas, M. D. Anderson Cancer Center, 1515 Holcombe Boulevard, Houston, Texas 77030, USA e-mail: rrb@mdanderson

For Nature's policy on sharing of biological materials, see http://tinyurl.com/33fg2r

\section{Invasion biology is a discipline that's too young to die}

SIR - Mark Davis suggests in his book Invasion Biology that the discipline should be reabsorbed into general community ecology, as Emma Marris reports in her Review 'The end of the invasion?' (Nature 459, 327-328; 2009). In fact, invasion biology has a much wider remit than the management of a few highly invasive species. The comparison of alien and native species has contributed to a better understanding of population dynamics, ecosystem function and species evolution. The field still has much to teach us about biodiversity and ecological processes in general, and its relevance to conservation policy is growing.

The acceleration of international trade and travel has led to progressive mixing of biota from across the world, introducing increasing numbers of species to new regions. Although our knowledge of the drivers and consequences of these invasions is still poor, the wisdom of distinguishing species in relation to their geographical origin is confirmed by discoveries of the often marked difference between alien and native species in behaviour, traits and impact (see, for example, P. E. Hulme Global Ecol. Biogeogr. 17, 641-647; 2008). However, the position of invasion biology as a separate discipline will continue to be questioned until we have a better framework for understanding the impacts of invasive species, as well as for risk assessment and strategies to deal with them.

Plurality of discourse is, of course, valuable in any science, and Davis's book should help to instil a balanced perspective among scholars in a young field that is still defining its own unique contribution to ecological understanding.

Petr Pyšek Institute of Botany, Academy of Sciences of the Czech Republic, Zámek 1, 25243 Průhonice, Czech Republic e-mail: pysek@ibot.cas.cz Philip E. Hulme Bio-Protection Research Centre, PO Box 84, Lincoln University 7647, Canterbury, New Zealand

\section{For cancer, seek and destroy or live and let live?}

SIR — In his Essay 'A change of strategy in the war on cancer' (Nature 459, 508-509; 2009), Robert Gatenby proposes that controlling a tumour's growth may be more efficient in the long term than trying to eradicate it. However, it could be some time before we can establish whether such a switch in treatment strategy is clinically beneficial.

To keep cancer in check, Gatenby suggests giving progressively lower doses of cytotoxic drugs, rather than the maximum-tolerated doses used in conventional chemotherapy to try to destroy the tumour.

In fact, oncologists have for several years been using daily low doses of drugs such as etoposide or cyclophosphamide to control tumours that do not respond to chemotherapy at higher doses, an approach called metronomic therapy. Robert Kerbel and Judah Folkman and their colleagues discovered the potential of metronomic chemotherapy for restricting tumour blood-vessel growth some ten years ago (see, for example, G. Klement et al. J. Clin. Invest. 105, R15-R24; 2000) and Isaiah Fidler and Lee Ellis have proposed that cancer is a chronic disease and should be treated as such (I. J. Fidler and L. M. Ellis Nature Med. 6, 500-502; 2000).

But despite some promising results in adult and paediatric oncology, this approach remains marginal and limited to patients in relapse, or to those for whom conventional treatment has failed. Perhaps this is because a metronomic strategy challenges the predicted success of conventional regimens based on dose intensity.

Paradoxically, many oncologists have adopted metronomic scheduling with blockbuster drugs such as Gleevec, Tarceva and Sutent - targeted therapies administered daily at low doses even though no one really knows whether a classical regimen of higher doses every three weeks might prove more efficient.

Gatenby's idea of 'adaptative therapy', derived largely from mathematical modelling, is a tour de force. He may be paving the way for advocates of traditional chemotherapy, targeted therapies and metronomic strategies to work together in the patient's best interest. We hope that stakeholders will be prepared to test Gatenby's approach and to revisit the idea of treating cancer as a chronic disease.

Nicolas André Department of Paediatric Oncology, Hôpital pour Enfants de la Timone, Bd Jean Moulin, 13385 Marseille Cedex 5, France e-mail: nicolas.andre@ap-hm.fr Eddy Pasquier Children's Cancer Institute Australia for Medical Research, Randwick, New South Wales 2031, Australia

Readers are welcome to comment on this topic at http://tinyurl.com/n4jn45 